\title{
Path Design and Control Algorithms for Articulated Mobile Robots
}

\author{
Ulf Andersson, Kent Mrozek \\ Q Navigator AB \\ Aurorum 21B \\ SE-977 75 Luleå, Sweden \\ Kalevi Hyyppä \\ Div. of Industrial Electronics \\ Luleå University of Technology \\ SE-971 87 Luleå, Sweden
}

\author{
Kalle Åström \\ Dept. of Mathematics \\ Lund Institute of Technology \\ Box 118 \\ SE-221 00 Lund, Sweden
}

\begin{abstract}
Experiments have shown that continuity of curvature of a reference path and of its derivative with respect to distance are particularly important for articulated mobile robots. The X4Y4 curve and a control algorithm for an articulated mobile robot following an X4Y4 curve are presented. Experiments with a 55 ton LHD vehicle in an underground mine have shown that the sideways repeatability when driving through a $106^{\circ}$ curve at $2.8 \mathrm{~m} / \mathrm{s}$ is within $\pm 100 \mathrm{~mm}$, and when driving through a $10^{\circ}$ curve at $5.1 \mathrm{~m} / \mathrm{s}$ is within $\pm 50 \mathrm{~mm}$.
\end{abstract}

\section{Introduction}

Path design is an important issue for real-world mobile robots. Ill-designed curves on a path can cause large guidance errors, and also result in high stress on servos, motors, bearings etc. in the robot.

Traditionally reference paths have been designed from lines and arcs, laid out to make the heading along the path continuous. However it has been shown [Nelson and Cox, 1988] that it is not possible for most mobile robots to follow this kind of paths without errors.

The cause of errors is the discontinuity in curvature at the junctions between the lines and the arcs. A step-wise change of curvature demands infinite acceleration of the actuator controlling the curvature taken by the robot guide point. (The only exception is a tricycle-type mobile robot with the guide point placed under the steering wheel. This placement should however be avoided because of the resulting complex control of the orientation of the robot, and the wide swept area. The common placement of guide point is at the mid-point between the non-steered wheels for a tricycle-type mobile robot, and at the mid-point between the rear or front wheels of an articulated mobile robot.)

The importance of continuous curvature has been discussed extensively in [Nelson, 1989] where quintic polynomial paths are proposed, and in [Kanayama and. Hartman, 1989] where cubic spiral paths are proposed. In the second paper a smoothness cost function is introduced which makes it possible to find the optimally smooth path. In [Graettinger and Krogh, 1989] time-scaling has been proposed to fulfill the dynamic constraints of a path.

In none of these papers has continuity of derivative of curvature with respect to distance along the path been discussed. A step change of the curvature derivative results in a step in actuator torque in the steering mechanism of the mobile robot. This might be acceptable for non-articulated types but our experiments have shown that it is not acceptable for an articulated vehicle driving at high speed.

A rather different approach has been taken in [Steer, 1989] where the author proposes a gaussian shape of the steer angle versus time function of a tricycle-type mobile robot when driving in a curve. The gaussian function is infinitely differentiable, solving the problem with steps in actuator torque, but the path is complicated to find. In [Pin and Vasseur, 1990] a simple algorithm is presented to find the shortest path between two postures. Limitations of the steering rate is discussed.

In this paper we present and discuss the $X 4 Y 4$ curve. It consists of two or three parts depending on the total heading change along the curve. The first part is called the closing part and the last part (second or third) is called the opening part. They are both characterized by

$$
x^{4}+y^{4}=c^{4}
$$

where $x$ and $y$ are the coordinates of the path in a global coordinate system and $c$ is a positive scaling constant which 
controls the size of the curve. In the closing part the curvature goes from 0 to a finite value, and in the opening part the curvature returns from the same finite value to 0 . The closing and opening parts are actually mirrored versions of each other: the $X 4 Y 4$ curve is symmetric.

If the total heading change is $>90^{\circ}$ the $X 4 Y 4$ curve has a circular segment between the closing and opening parts. The radius of the circular segment is chosen such that the curvature is continuous along the whole $X 4 Y 4$ curve.

The guidance controller on board the vehicle calculates set values to a servo which controls the articulation angle of the vehicle from measured errors in distance and angle. The distance error is defined as the distance from the guide point on the vehicle to the nearest point on the path, and the angle error as the angle between the heading of the vehicle guide point and the tangent to the path at the nearest point.

The calculation of the nearest point on the closing and opening parts of an X4Y4 curve can only be done numerically whereas the nearest point on the circular segment of an $X 2 Y 2$ curve can be expressed in closed form.

To increase the performance of a guidance controller when driving the vehicle along an X4Y4 curve it is favorable to include a feed-forward part in the controller. This part is designed to be a function of the curvature at the nearest point. The function is dependent on the vehicle geometry.

The X4Y4 curve has been implemented on a navigation and guidance system on an articulated 55 ton mining vehicle driving at $5.3 \mathrm{~m} / \mathrm{s}$. Most of the development work was done using our test vehicle CALMAN [Larsson et al., 1994] which is a modified articulated lawn-mover of maximum speed $2.5 \mathrm{~m} / \mathrm{s}$. Figure 1 shows a photograph of CALMAN.

The navigation system onboard CALMAN is based on a laser scanner which measures headings to retroreflective beacons at known positions in the workspace. This scanner is visible in Figure 1 mounted on top of one of the boxes which houses the control system. The navigation system was developed at Luleå University of Technology [Нyyppä, 1986; 1989; 1993; 1994; Hyyppä et al., 1994; Wiklund et al., 1988]. It has been produced commercially for several years.

\section{Properties of the $X 4 Y 4$ curve}

\subsection{Desirable Path Properties:}

- Continuous curvature $K(s)$. The variable $s$ is the distance along the path

- Continuous curvature change with distance $d K / d s$.

\subsection{The X4Y4 curve}

The $X 4 Y 4$ curve is a symmetric curve consisting of three parts if the total heading change is more than $90^{\circ}$ or of two parts if the total heading change is less than or equal to $90^{\circ}$. To make the curvature $K(s)$ continuous it is chosen to have the same value on both parts at the junction(s).

\section{Total Heading Change $>90^{\circ}$}

The first part is the closing $X 4 Y 4$ characterized by:

- $\quad$ heading change $=45^{\circ}$.

- $K=d K / d s=0$ at the start point.

- $K \neq 0$ at the end point.

- $d K / d s=0$ at the end point.

The middle part is a circular arc (X2Y2) characterized by:

- $K=$ constant.

- $d K / d s=0$

The last part is the opening $X 4 Y 4$ characterized by:

- $\quad$ heading change $=45^{\circ}$.

- $K=d K / d s=0$ at the end point.

- $K \neq 0$ at the start point.

- $d K / d s=0$ at the start point.

Total Heading Change $=90^{\circ}$

As above but without the middle part (X2Y2).

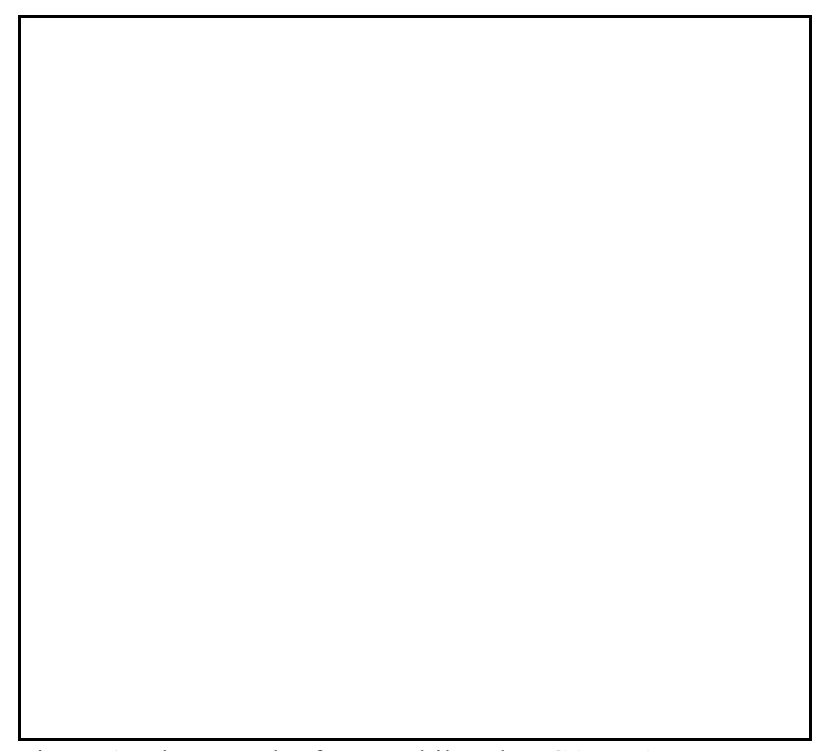

Figure 1. Photograph of our mobile robot CALMAN.

Total Heading Change $<\mathbf{9 0}^{\circ}$

Only closing $X 4 Y 4$ and opening $X 4 Y 4$ parts with less than $45^{\circ}$ heading change in both. The curvature is still continuous but at the joining point we get

$$
\left(\frac{d K}{d s}\right)_{\text {opening part }}=-\left(\frac{d K}{d s}\right)_{\text {closing part }}
$$

i.e. the curvature derivative with respect to distance is discontinuous. An alternative design is to include a small $X 2 Y 2$ part in the middle which instead of one jump gives two smaller jumps in $d K / d s$. 
Examples of four alternatives for designing a $90^{\circ}$ curve are shown in Figure 2. Figure 3 shows that only the fourth alternative gives the desirable property of continuous $d K / d s$ along the whole curve.

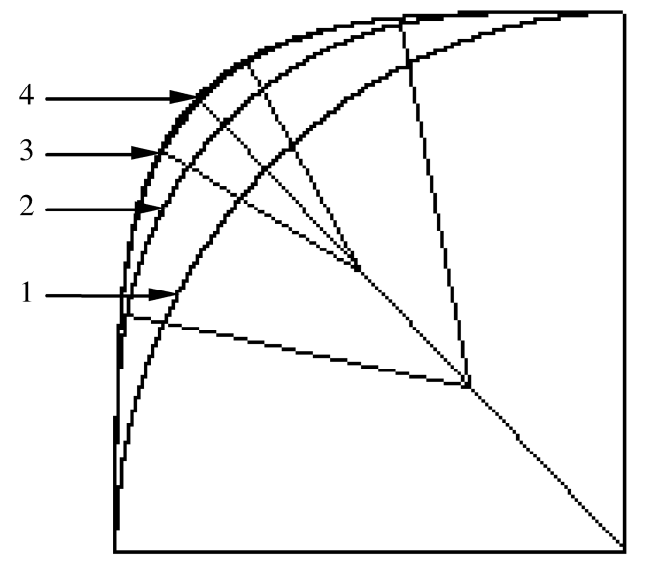

Figure $2.90^{\circ}$ curve examples.

1. $X 2 Y 2\left(90^{\circ}\right)$

2. $X 4 Y 4\left(11.45^{\circ}\right)-X 2 Y 2\left(67.1^{\circ}\right)-X 4 Y 4\left(11.45^{\circ}\right)$

3. $X 4 Y 4\left(30^{\circ}\right)-X 2 Y 2\left(30^{\circ}\right)-X 4 Y 4\left(30^{\circ}\right)$

4. $X 4 Y 4\left(45^{\circ}\right)-X 4 Y 4\left(45^{\circ}\right)$

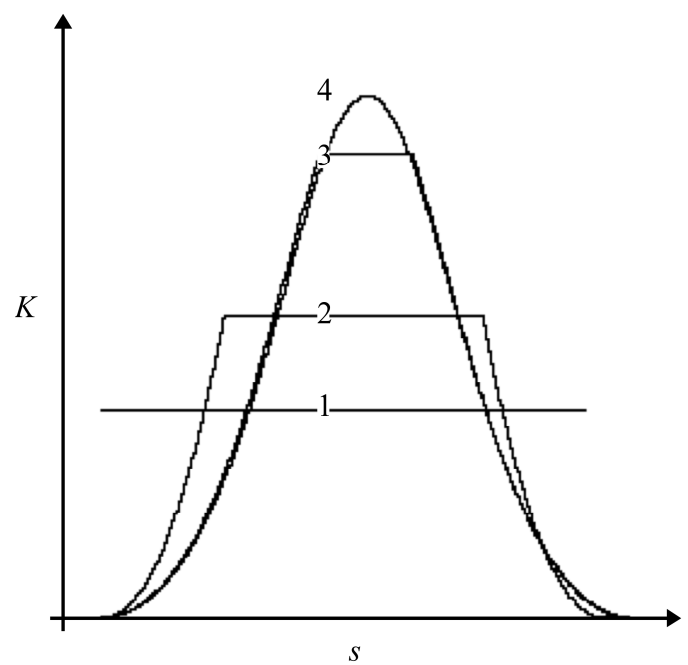

Figure 3. Curvatures $K(s)$ from $90^{\circ}$ curve examples. The symbol $s$ denotes the distance along the curve.
1. $\mathrm{X} 2 \mathrm{Y} 2\left(90^{\circ}\right)$
2. $\mathrm{X} 4 \mathrm{Y} 4\left(11.45^{\circ}\right)-\mathrm{X} 2 \mathrm{Y} 2\left(67.1^{\circ}\right)-\mathrm{X} 4 \mathrm{Y} 4\left(11.45^{\circ}\right)$
3. $\mathrm{X} 4 \mathrm{Y} 4\left(30^{\circ}\right)-\mathrm{X} 2 \mathrm{Y} 2\left(30^{\circ}\right)-\mathrm{X} 4 \mathrm{Y} 4\left(30^{\circ}\right)$
4. $\mathrm{X} 4 \mathrm{Y} 4\left(45^{\circ}\right)-\mathrm{X} 4 \mathrm{Y} 4\left(45^{\circ}\right)$

\section{Controller for the X4Y4 curve}

A block diagram of our proposed control system for articulated mobile robots is shown in Figure 4. The set value for the articulation angle $\alpha_{\text {set }}$ is calculated according to

$$
\alpha_{s e t}=f\left(d_{p a t h}, \Delta_{p a t h}\right)+F F\left(K_{n}\right)
$$

where

$d_{\text {path }}$ is the sidewise error to the reference path, the sign is positive when the guide point is to the left of the curve, and negative when it is to the right of the curve;

$\Delta_{\text {path }}$ is the heading error to the reference path and

$$
-\pi \leq \Delta_{\text {path }} \leq \pi
$$

$K_{n}$ is the curvature at the nearest point on the reference path;

$f$ is the error controller function (there are a number of standard controller structures to choose among);

$F F$ is a feed-forward function of $K_{n}$ which depends on vehicle geometry, rotation direction of the curve (clockwise or counter-clockwise), and the driving direction of the robot (forward or reverse).

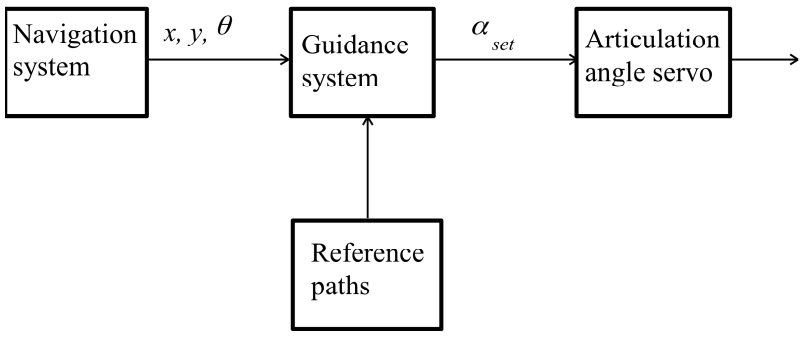

Figure 4. Block description of the proposed control system for articulated mobile robots.

\subsection{Steps in Determining $d_{p a t h}, \Delta_{\text {path }}$ and $K_{n}$ on the X4Y4 part.}

It is assumed that the position and heading $(x, y, \theta)$ of the guide point in the global co-ordinate system is determined by a navigation system.

A normalised co-ordinate system is used as an aid in determining the variables $d_{\text {path }}, \Delta_{\text {path }}$ and $K_{n}$. Figure 5 shows an $X 4 Y 4$ part giving a heading change of $45^{\circ}$ in normalised form, together with some variables which are used for the calculations. 


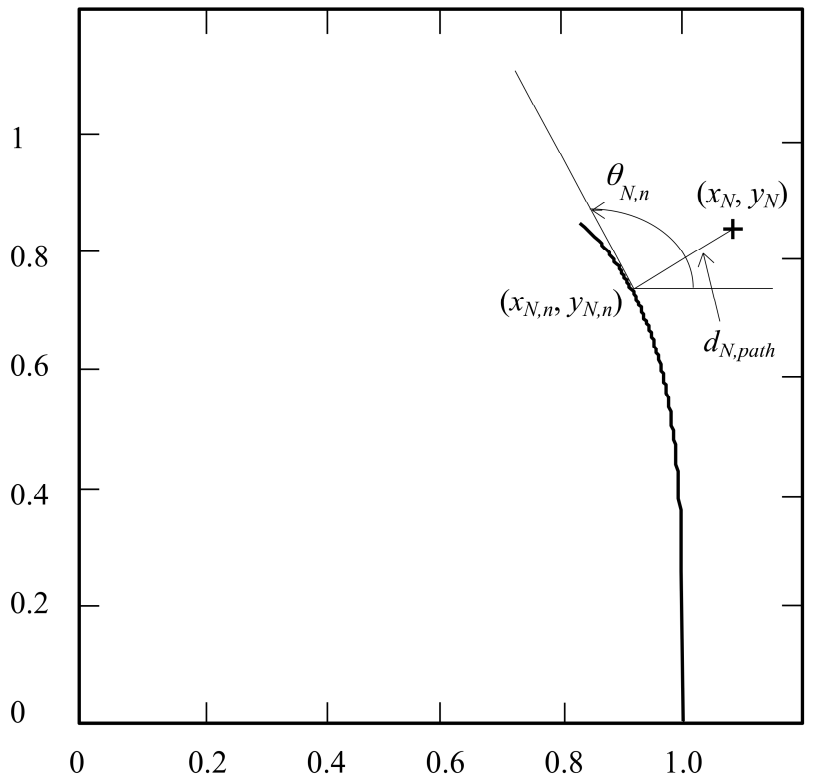

Figure 5. Normalised co-ordinate system of the $X 4 Y 4$ part with a heading change of $45^{\circ}$. The symbol ' + ' denotes the position of the mobile robot. Subscript $N$ denotes 'normalised' and subscript $n$ denotes the nearest point on the curve.

The steps to calculate $d_{\text {path }}, \Delta_{\text {path }}$ and $K_{n}$ are:

1. Transform the $X 4 Y 4$ part and the global position $(x, y)$ of the mobile robot guide point to the normalised coordinate system.

2. Use a numerical method, e.g. Newton-Raphson, to find the nearest point on the X4Y4 part. Use appropriate start values.

3. Calculate the heading of the curve, i.e. the direction of the tangent, and the curvature at the nearest point.

4. Transform from the normalised co-ordinate system back to the global co-ordinate system i.e.

$$
\left(x_{N, n}, y_{N, n}, \theta_{N, n}, K_{N, n}\right) \rightarrow\left(x_{n}, y_{n}, \theta_{n}, K_{n}\right) .
$$

5. Calculate the guidance controller inputs.

$$
\begin{gathered}
\left|d_{\text {path }}\right|=\sqrt{\left(x-x_{n}\right)^{2}+\left(y-y_{n}\right)^{2}} \\
\Delta_{\text {path }}=\theta-\theta_{n}
\end{gathered}
$$

\section{Design of $\mathrm{X} 4 \mathrm{Y} 4$ curves}

The design of the curves need to take into account the limitations of the vehicle which are:

- Maximum articulation angle.

- Maximum articulation angle speed.

The maximum articulation angle and the geometry of the vehicle limit the maximum curvature the vehicle can follow, which gives a maximum allowed curvature $K_{\max }$.
The curvature speed $K_{v}$ (i.e. the time derivative of the curvature for the vehicle when driving along a curve with a certain speed $d s / d t$ ) can be expressed as

$$
K_{v}=\frac{d K}{d t}=\frac{d K}{d s} \cdot \frac{d s}{d t}
$$

The vehicle should drive with constant speed along the curve to get a smooth ride. This condition together with the maximum articulation angle speed and the geometry of the vehicle, limits $K_{v}$ to $K_{v \max }$.

The limits $K_{\max }$ and $K_{v \max }$ should contain some safety margins.

\subsection{A Design Example}

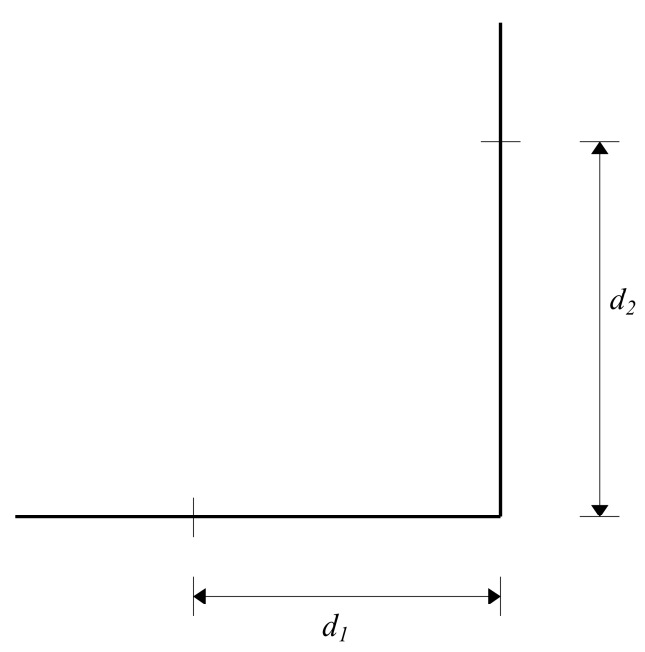

Figure 6. The basis for the path design is a polygon of straight lines, which could represent the centre lines for tunnels in a mine. In this example a curve should be designed within the limits defined by $d_{1}$ and $d_{2}$. If $d_{1}<d_{2}$ then $d_{1}$ is the limit (and then also the scale factor $\mathrm{c}=d_{l}$ in case of a $90^{\circ}$ curve).

The design steps are:

1. Make a curve with the smallest possible curvature within the size limits.

2. Check that the maximum curvature does not imply an articulation angle greater than the limit of the vehicle.

3. If the maximum curvature speed implies an articulating angle speed of the vehicle greater than the limit of the vehicle, reduce the vehicle speed.

\section{Experiences of the $\mathrm{X} 4 \mathrm{Y} 4$ curve}

\subsection{Non-articulated Vehicles}


The X4Y4 curve has for some time been used successfully in a number of AGV installations, and is commercially available. The vehicle types have been:

- Tricycle vehicles (single steer and drive unit).

- Diff vehicles (differential drives).

- Quad vehicles (dual steer and drive units).

The maximum speed of these AGV's is typically $1.0 \mathrm{~m} / \mathrm{s}$.

\subsection{Articulated Vehicles}

Lately, the curve has also been used for the following articulated vehicles:

- CALMAN, our modified $200 \mathrm{~kg}$ articulated lawn mover with maximum speed $=2.5 \mathrm{~m} / \mathrm{s}$.

- A 55 ton LHD vehicle with maximum speed $=5.3$ $\mathrm{m} / \mathrm{s}$.

The main difference between AGV's and articulated vehicles is that the chassis parts do not rotate relative to each other when an AGV turns.

This difference becomes very clear in practice if there is a discontinuity in $d K / d s$. In an $\mathrm{AGV}$, limited discontinuities is not noticeable due to the stiff chassis and the low speed. In an articulated vehicle, even small discontinuities in $d K / d s$ becomes noticeable in the performance of the vehicle due to the two part chassis and the high speed.

\section{CALMAN}

Our mobile robot CALMAN has been used in several research projects [Larsson et al., 1994].

The control of the articulation joint is done with a DCmotor connected to the joint via spring tensioned wires. Due to the springs, the connection is not very stiff which requires high damping in the servo for the articulation angle.

\section{The LHD Vehicle}

The LHD (Load Haulage and Dump) vehicle is used for transporting rock in its bucket in underground mines. To our knowledge automatic (i. e. driverless) LHD vehicles have not yet been used commercially, although experiments with LHD vehicles following an optical guide line have been reported [Hurteau et al., 1992].

The LHD vehicle we have used has the following limitations:

- Maximum steering angle $33^{\circ}$.

- Maximum steering angle speed $18^{\circ} / \mathrm{s}$.

- Electrically driven (1000 V, 3-phase AC) with 4 gear positions. Approximate speeds for the gear positions are:

- $1: 1.0 \mathrm{~m} / \mathrm{s}$.

- 2: $1.7 \mathrm{~m} / \mathrm{s}$.

- $\quad 3: 2.8 \mathrm{~m} / \mathrm{s}$.

- $4: 5.1 \mathrm{~m} / \mathrm{s}$.
The control of the articulation angle is done via a hydraulic system. The electrical interface to the hydraulic system has electrical proportional valves which are controlled by PWM-signals from the control system.

\section{The Path Layout for the LHD Vehicle}

The layout (see Figure 7) consisted of straight-line segments and curve segments of the X4Y4 type. The straight-line segments were connected with the four curves $1,2,3$ and 4 in Figure 7.

Curves 2 and 4 were designed to have a safety margin of approximately $5^{\circ}$ in articulation angle and approximately $7 \%$ in articulation angle speed. The design was made for a constant speed of the vehicle of $2.8 \mathrm{~m} / \mathrm{s}$ along the curve.

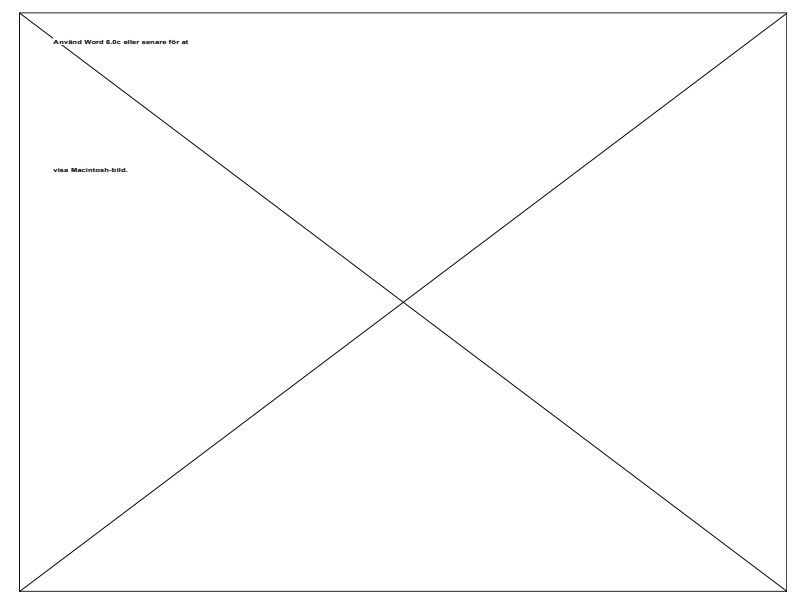

Figure 7. Layout for the 55 ton articulated LHD vehicle in an underground mine. The symbol $\mathrm{X}$ marks the position of the power supply unit for the vehicle. The $\mathrm{x}$-axis of the global co-ordinate system points to the right in the figure. The distance, in the $\mathrm{x}-$ direction, from the supply unit to curve 4 is approximately $140 \mathrm{~m}$. The curves are of the following types:

1. $X 4 Y 4\left(8^{\circ}\right)-X 4 Y 4\left(8^{\circ}\right)$

2. $X 4 Y 4\left(45^{\circ}\right)-X 2 Y 2\left(16^{\circ}\right)-X 4 Y 4\left(45^{\circ}\right)$

3. $X 4 Y 4\left(2.5^{\circ}\right)-X 2 Y 2\left(5.5^{\circ}\right)-X 4 Y 4\left(2.5^{\circ}\right)$

4. $X 4 Y 4\left(45^{\circ}\right)-X 2 Y 2\left(5.5^{\circ}\right)-X 4 Y 4\left(45^{\circ}\right)$

Curves 1 and 3 have heading changes less than $90^{\circ}$ and therefor have discontinuities in $d K / d s$ which made a "trial and error" design necessary. The designed speed for these curves is high $(5.1 \mathrm{~m} / \mathrm{s})$ which demanded some fine tuning to give a satisfactory result.

\section{Experimental Results}

The logged position of the vehicle when driving through curve 4 is shown in Figure 8. The drag from the power supply cable influenced the path taken by the vehicle somewhat. When driving into the curve the drag was considerably higher than when driving out. The drag creates a torque at the articulation joint which tries to decrease the articulation angle.

The path in the forward direction (left to right in Figure 7) is slightly uphill. Therefor the speed was somewhat lower 
when driving in the forward direction compared to when driving in the reverse direction. The maximum speed (5.3 $\mathrm{m} / \mathrm{s}$ ) was reached in the beginning of curve 1 when driving in the reverse direction. The logged speed of the vehicle when driving through curve 4 is shown in Figure 9. The speed was sampled with a rate of 40 samples/s.

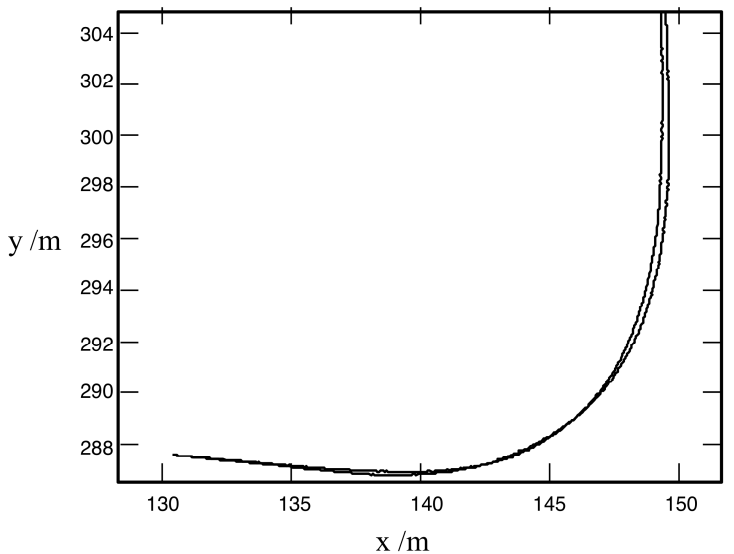

Figure 8. Logged position when running in and out of curve 4 . The electric power supply cable mounted in the rear of the LHD vehicle introduced a force causing the LHD vehicle to take an "outer" curve when running in.

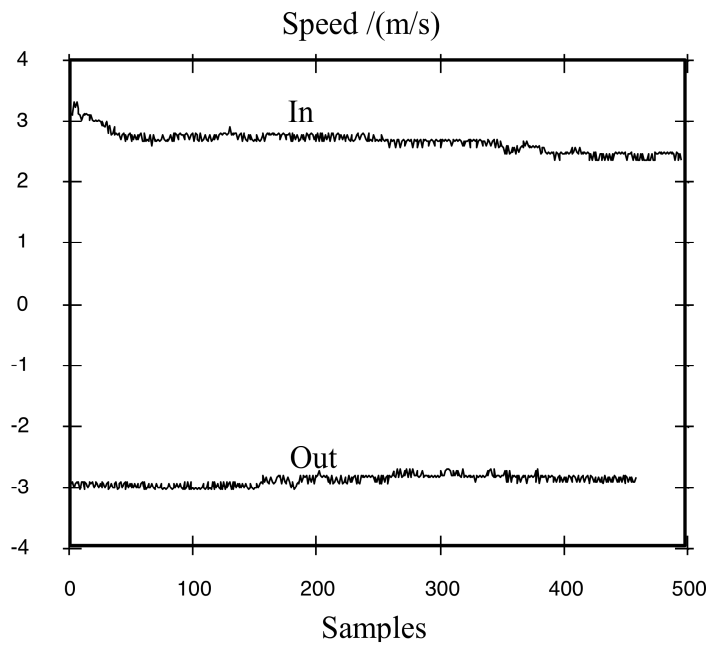

Figure 9. Logged speed $(\mathrm{m} / \mathrm{s})$ when driving in and out of curve 4 . Sampling frequency $=40 \mathrm{~Hz}$. Speed is positive when driving in and negative when driving out. It is slightly uphill when going in which explains the lower speed in that direction.

The logged articulation angles of the vehicle when driving in and out of curve 4 are shown in Figure 10 and when driving twice through curve 4 are shown in Figure 11.

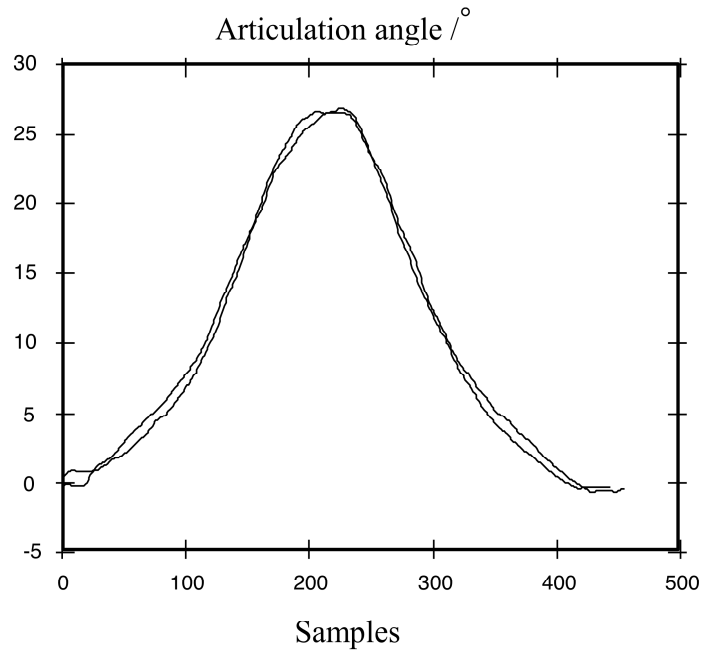

Figure 10. Logged articulation angle when running in and out of curve 4.

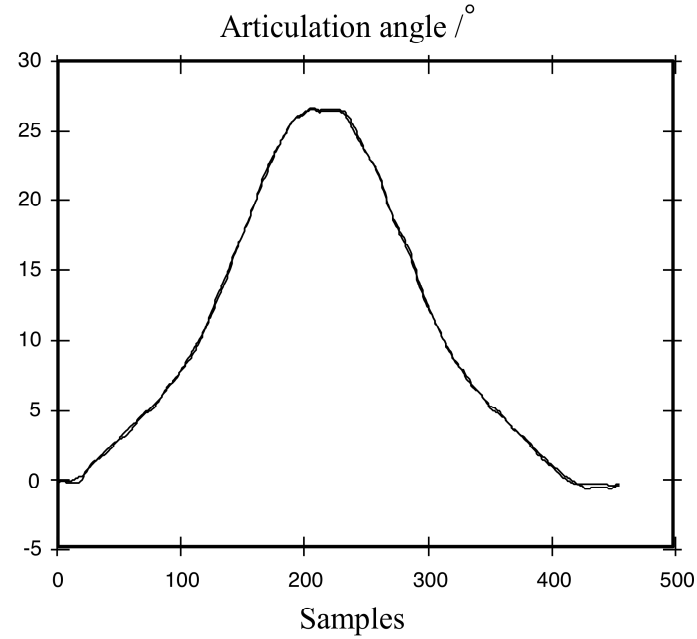

Figure 11. Logged articulation angle for two runs into curve 4 . The small differences illustrates the high repeatability of the system.

Finally, the logged articulation angle of the vehicle when driving through curve 3 is shown in Figure 12. This curve has an $X 2 Y 2$ part which is visible in the figure as an almost constant articulation angle for the middle part of the curve. 


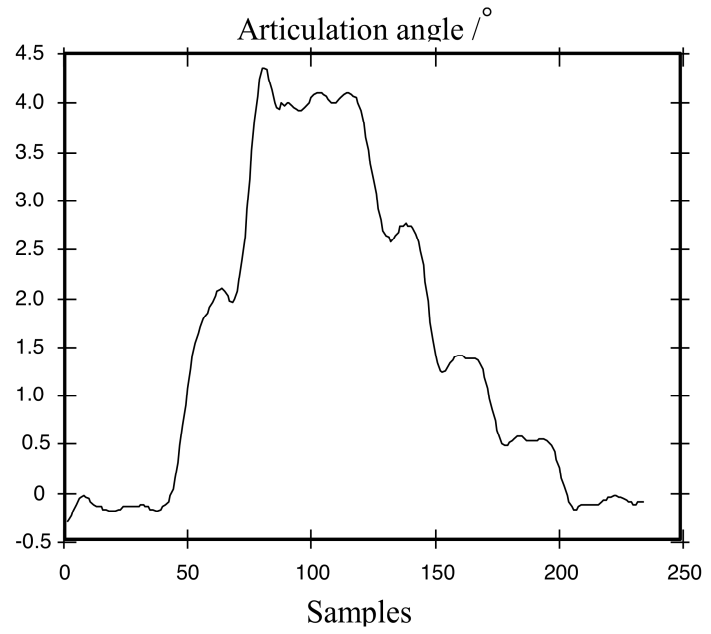

Figure 12. Logged articulation angle when driving into curve 3. The $5.5^{\circ} X 2 Y 2$ part in the middle shows up as an almost constant articulation angle between samples 80 and 130 .

\section{Conclusions}

X4Y4 curves without discontinuities in $d K / d s$ give excellent path-following performance of an articulated vehicle. The design does not require any trial and error. Practical tests have shown that safety margins of $\approx 5^{\circ}$ for $K$ and of $\approx 40 \%$ for $K_{v}$ make it possible to keep the guidance controller nonsaturated in the curves.

X4Y4 curves with discontinuities (less than $90^{\circ}$ curves) are possible for articulated vehicles, but demand a trial and error based design when driving at high speed.

After a large number of runs in the mine we have come to the conclusion that the repeatability in curve 2 and 4 is within $\pm 100 \mathrm{~mm}$. The repeatability on straight paths and the wider curves (curve 1 and 3 ) is within $\pm 50 \mathrm{~mm}$.

Curve 1 and 3 have a small discontinuity in $d K / d s$. The discontinuity is so small that it is not noticeable in practice. However, some care had to be exercised when deciding the scale factor $c$ for these curves. There were lower limits for the two scale factors. Going below these limits caused the guidance controller to go into saturation when driving in 4:th gear.

\section{References}

[Graettinger and Krogh, 1989] T. J. Graettinger and B. H. Krogh, "Evaluation and Time-Scaling of Trajectories for Wheeled Mobile Robots", Trans. Of the ASME, vol. 111, pp. 222-231, June 1989.

[Hurteau et al., 1992] R. Hurteau et al., "Optical Guidance System for Underground Mine Vehicles", Proc. Of the 1992 IEEE Int. Conf. On Robotics and Automation, pp. 639-644, Nice, France, May 1992.

[Hyyppä, 1986] K. Hyyppä, “Optical navigation system using passive identical beacons", Proc. Intelligent Autonomous Systems, Amsterdam, December 1986.
[Hyyppä, 1989] K. Hyyppä, "Luleå Turbo Turtle (LTT)”, Proc. IEEE/RSJ International Workshop on Intelligent Robots and Systems '89, Tsukuba, September 1989.

[Hyyppä, 1993] K. Hyyppä," On a laser anglemeter for mobile robot navigation", Doctoral Thesis 1993:117D, Luleå University of Technology 1993.

[Hyyppä, 1994] K. Hyyppä, "Design considerations in a laser navigation system for mobile robots", Mechatronics, vol. 4, no. 2, pp. 199-206, 1994.

[Hyyppä et al., 1994] K. Hyyppä, U. Wiklund, U. Andersson, A. Wernersson, T. Gustafsson, G. Hillerström, and C. Zell, "Navigational experiments with the autonomous mobile robot LTT using angle measurements to identical beacons", Tampere Int. Conf. on Machine Automation, Tampere, February 1994.

[Kanayama and. Hartman, 1989] Y. Kanayama and B. I. Hartman, "Smooth Local Path Planning for Autonomous Vehicles", 1989 IEEE Int. Conf. on Robotics and Automation, pp. 1264-1270, Scottsdale, Arizona, May 1989.

[Larsson et al., 1994] U. Larsson, C. Zell, K. Hyyppä and Å. Wernersson, "Navigating an Articulated Vehicle and Reversing with a Trailer", 1994 IEEE Int. Conf. on Robotics and Automation, San Diego, May 1994.

[Nelson, 1989] W. L. Nelson, "Continuous SteeringFunction Control of Robot Carts", IEEE Trans. On Industrial Electronics, vol. 36, no. 3, pp. 330-337, August 1989.

[Nelson and Cox, 1988] W. L. Nelson and I. J. Cox, "Local Path Control for an Autonomous Vehicle", 1988 IEEE Int. Conf. on Robotics and Automation, pp. 1504-1510, Philadelphia, Pennsylvania, April 1988.

[Pin and Vasseur, 1990] F. G. Pin and H. A. Vasseur, "Autonomous Trajectory Generation for Mobile Robots with Non-Holonomic and Steering Angle Constraints", Proc. IEEE Int. Workshop on Intelligent Motion Control, pp. 20-22, Istanbul, August 1990.

[Steer, 1989] B. Steer, "Trajectory Planning for a Mobile Robot", The Int. Journal of Robotic Research, vol. 8, no. 5, pp. 3-14, October 1989.

[Wiklund et al., 1988] U. Wiklund, U. Andersson and K. Hyyppä, "AGV navigation by angle measurements", Proc. 6th int. Conf. Automated Guided Vehicle System, pp. 199-212, Brussels, October 1988. 